\title{
Acute Q Fever Presenting with Multi-Organ Failure: Re-Evaluation of the Initial Diagnosis
}

\author{
Noel Lorenzo Villalba, Maria Belen Alonso Ortiz, Laura Ros Sanjuan, Jerónimo Artiles Vizcaíno, Saturnino Suárez Ortega \\ Hospital Universitario de Gran Canaria Doctor Negrín, Las Palmas de Gran Canaria, Spain
}

\section{Doi: 10.12890/2016_000423- European Journal of Case Reports in Internal Medicine - ๑ EFIM 2016}

Received: 09/03/2016

Accepted: $18 / 04 / 2016$

Published: $25 / 05 / 2016$

How to cite this article: Lorenzo N, Alonso MB, Ros L, Artiles J, Suárez S. Acute Q fever presenting with multi-organ failure: re-evaluation of the initial diagnosis. EJCRIM 2016;3:doi:10.12890/2016_000423

Conflicts of Interests: The Authors declare that there are no competing interests.

This article is licensed under a Commons Attribution Non-Commercial 4.0 License

\section{ABSTRACT}

We present the case of a 48-year-old man admitted to the critical care unit with atrial fibrillation, and acute heart and kidney failure accompanied by coagulopathy and an abnormal liver test. Initially diagnosed as a non-ST elevation myocardial infarction, re-evaluation of the case led to the consideration of severe sepsis. Q fever and leptospirosis were the most probable causes and empiric treatment was initiated. A complete recovery was achieved following treatment.

\section{LEARNING POINTS}

- Epidemiological antecedents of interest must always be collected in the clinical record.

- Acute Q fever infection may be mild or present with multiple organ damage.

- Empiric treatment with doxycycline must be started when Q fever is suspected.

\section{KEYWORDS}

Raw milk, multiorgan failure, Q fever

\section{INTRODUCTION}

Q fever is a widespread zoonotic infection caused by the pathogen, Coxiella burnetii, that has both acute and chronic manifestations ${ }^{[1]}$. Patients with Q fever present with a wide spectrum of disease manifestations. In some patients, the clinical signs of Q fever are often mild or absent ${ }^{[2]}$, while others present with symptoms of acute or chronic infection ${ }^{[1]}$. Symptomatic infection is more likely to occur in adults ${ }^{[2]}$.

\section{CASE DESCRIPTION}

A 48-year-old man with no relevant antecedents was admitted to the critical care unit because of new onset atrial fibrillation, and acute heart and renal failure. Laboratory tests showed elevation of troponin T, creatine-kinase and liver enzymes as well as metabolic acidosis with an elevated anion gap and coagulopathy. Chest x-rays revealed signs of cardiomegaly and vascular redistribution (Fig. 1), while ECG demonstrated atrial fibrillation with T-wave inverted from V2 to V4. An echocardiography was immediately performed and showed severe left ventricular dysfunction with no valvulopathy or pericardial effusion. In this context, non-ST elevation myocardial infarction was diagnosed and the patient was transferred to the cardiology ward. As no clinical or laboratory improvement had been achieved by day 4 of 


\section{of Case Reports in \\ Internal Medicine}

admission, an internists was consulted for advice. An interview with the patient revealed he had experienced cough, yellow expectoration and chills for 20 days before admission, and so he was prescribed a 10-day course of amoxycillin. After completion of the antibiotics, the patient complained of epigastric abdominal pain, nausea and vomiting which were assumed to be due to the amoxycillin and so metoclopramide was prescribed. However, the patient continued to feel weak and complained of myalgia, shortness of breath and oscillating fever, predominantly in the evening. The patient was a farmer from a rural area in Gran Canaria and consumes raw milk.

On examination, the patient was afebrile, blood pressure and heart rate were within the normal range but he was saturating $94 \%$ on room air. He was slightly jaundiced and cardio-pulmonary examination revealed arrhythmic heart sounds and rales in both pulmonary bases. Laboratory tests showed a marked leucocytosis, acute renal failure with a pattern compatible with acute tubular necrosis, and elevated liver enzymes. Prothrombin time was 34\% (normal value $>70 \%$ ) and both creatine-kinase and troponin T were elevated. In light of the patient's history and laboratory tests, sepsis of unknown origin was considered. The overall clinical and biological picture suggested that leptospirosis and Q fever were the most probable causes. Therefore, $100 \mathrm{mg}$ of doxycycline twice daily was initiated and the patient was transferred to the internal medicine ward. Blood samples were sent for confirmation of either leptospirosis or Q fever as well as to rule out other pathogens. Biological recovery of renal and liver function was obtained $48 \mathrm{~h}$ after antibiotic initiation, with declines also in creatine-kinase and troponin (Table 1). Chest x-rays showed a significant improvement compared to the previous study (Fig. 1). Serology, using an immunofluorescence assay (IFA), was compatible with recent Q fever infection as antiphase II was 512 for IgG and 112 for IgM. The polymerase chain reaction test for serum samples was not available in our hospital. The patient was released on day 15 after admission.

\begin{tabular}{|l|l|l|l|l|l|l|}
\hline Parameter & Admission & Day 2 & Day 4 & Day 6 & Day 8 & Discharge \\
\hline Leukocytes $\left(\times 10^{3} / \mathrm{ML}\right)$ & 21.80 & 20.13 & 16.10 & 11.00 & 11.50 & 9.3 \\
\hline Creatinine (mg/dL) & 2.13 & 1.95 & 1.54 & 1.19 & 1.14 & 1.06 \\
\hline CK (U/L) & 527 & 470 & 76 & & 25 & 34 \\
\hline TnT (ng/L) & 34.97 & 32.25 & 21.71 & 112.8 & 17.78 & 18.64 \\
\hline Total bilirubin (mg/dL) & 3.03 & 2.8 & 2.7 & 2.4 & 2 & 0.57 \\
\hline AST (U/L) & 650 & 1897 & 116 & 669 & 251 & 40 \\
\hline ALT (U/L) & 495 & 1268 & 1188 & 1058 & 624 & 40 \\
\hline GGT (U/L) & 174 & 150 & 96 & 65 & 50 & 51 \\
\hline Alkaline phosphatase & 190 & 188 & 150 & 133 & 99 & 88 \\
\hline LDH (U/L) & 400 & 350 & 270 & 200 & 170 & 135 \\
\hline PT (\%) & 34 & 43 & 54 & 115 & 90 & 90 \\
\hline Procalcitonin (ng/mL) & 0.3 & & 0.2 & & & \\
\hline
\end{tabular}

Table 1: Laboratory findings.

ALT, alanine aminotransferase; AST, aspartate aminotransferase; CK, creatine-kinase; GGT, $\gamma$-glutamyl transpeptidase; LDH, lactate dehydrogenase;

PT, prothrombin time as a percentage of normal (normal value > 70\%); TnT, troponin T.

\section{DISCUSSION}

The patient presented to the emergency department with a history of general malaise, fever and multi-organ failure. Upon arrival, cardiac enzymes were elevated but not showing a clear ascending curve in the context of acute renal failure, and were accompanied by new onset atrial fibrillation, initially suggesting non-ST myocardial infarction. However, this presumptive diagnosis did not explain the overall picture considering the absence of chest pain, and the presence of fever and elevated infectious markers. 

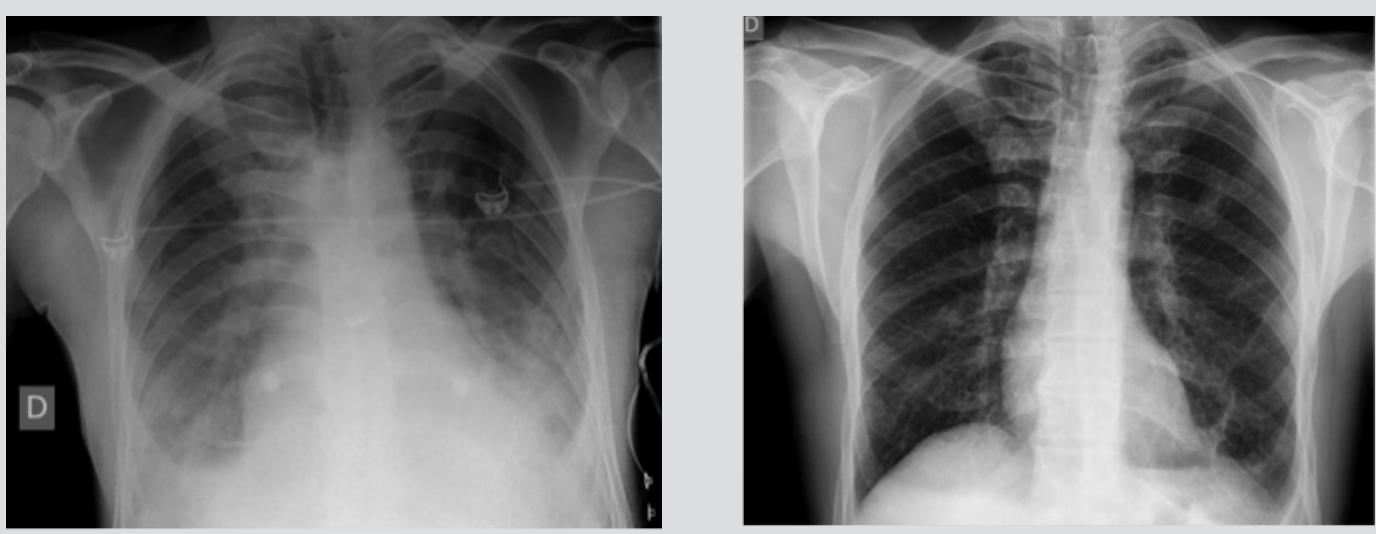

Figure 1 (Left) Bilateral pleural effusion and hilar congestion; (right) complete resolution.

On the other hand, an echocardiogram upon arrival showed global left ventricular dysfunction but no myocardial scars or localized myocardial injury. Atrial fibrillation with rapid ventricular response might explain the presence of inverted T waves. In contrast to the initial diagnosis, clinical and laboratory courses were not as expected or in accordance with the treatment, which caused doctors to doubt the diagnosis and therefore call on an internist for advice.

Upon patient re-evaluation, some elements in favour of sepsis were noted: history of fever, myocarditis, elevation of liver enzymes, coagulopathy, acute renal failure and marked leucocytosis. Many microbiological agents could have caused such a clinical picture, but as the patient came from a rural area with a high prevalence of both $\mathrm{Q}$ fever and leptospirosis, these were the main suspected aetiological agents. From the cardiovascular point of view, two elements help to distinguishing myocarditis from myocardial infarction: acute myocarditis diffusely affects the heart muscle, as in our case, whereas only a specific area is affected in myocardial infarction. Myocardial infarction also causes a focal scar which was not found on the echocardiogram performed before the patient's discharge. Myocarditis either completely heals or leads to diffuse damage compatible with congestive cardiomyopathy ${ }^{[1]}$. In our case, the findings on both echocardiography and chest $\mathrm{x}$-rays disappeared before completion of the cycle of antibiotics (doxycycline).

An important element to keep in mind in the case of our patient is that he was a farmer in close contact with goats and consumed raw milk. Two elements are involved in the transmission of $C$. burnetii, the causative agent of $Q$ fever, elements that are sometimes forgotten when examining the patient which can make the diagnosis difficult, as in our case. Everyday cases are sometimes misdiagnosed as viral infections, which consequently could then progress to chronic infections which are difficult to treat. Q fever may lead to serious complications and even death in patients with acute disease, especially those with meningoencephalitis or myocarditis, and more frequently in chronically infected patients with endocarditis ${ }^{[2]}$.

$\mathrm{Q}$ fever is a zoonosis with a worldwide distribution, New Zealand being the only exception ${ }^{[2]}$. In some countries $\mathrm{Q}$ fever is an occupational disease, but not in the Canary Islands where cases of human infection have been described in individuals not in contact with infected animals. Intermittent high-level shedding occurs in female animals at parturition, with millions of bacteria being released per gram of placenta; humans are usually infected by contaminated aerosols. In addition, bacteria can be carried by the wind far away from their original location to contaminate other animals. Because $\mathrm{Q}$ fever is rarely a notifiable disease, the incidence of human $\mathrm{Q}$ fever cannot be assessed in most countries. In areas of high Q fever prevalence like the Canary Islands, and in the presence of a clinical picture suggesting this entity, administration of antibiotics for 14 days is justified while the results of serological tests are awaited and even if the first tests are negative as seroconversion may be delayed.

The use of doxycycline generally results in a good clinical response as in our case, but in those with severe systemic or liver involvement, corticoids are sometimes required to reverse cholestasis. A biphasic $\mathrm{Q}$ fever has been described that mimics brucellosis in which fever reappears after the first episode has been appropriately treated. In chronic forms, hydroxychloroquine potentiates the intracellular microbiological effect ${ }^{[3]}$.

The diagnosis of acute $Q$ fever is based on the finding of antiphase II antibodies against $C$. burnetii using indirect immunofluorescence. IgG titres $\geq 1 / 200$, IgM titres $\geq 1 / 70$, or the seroconversion of samples collected during acute phases and convalescence ( $2-4$ weeks) are considered to be diagnostic ${ }^{[4]}$. 
As $C$. burnetii is highly infective and only one bacterium may cause the disease, another diagnostic tool could be polymerase chain reaction (PCR) but it is costly and not always available. In some Spanish hospitals, PCR determination costs $€ 60$ while immunofluoresence is widely available and only costs $€ 30$. Besides, PCR is reserved for specific areas such as the heart valves or the endocardium. The complement fixation technique has low sensibility and so is not used. Another diagnostic tool could be a liver biopsy to determine if the classic donut granulomas are present ${ }^{[5]}$. In our case, only indirect immunofluorescence was available and lgG titres of 1/512 and lgM titres of $1 / 112$ confirmed acute $Q$ fever infection.

\section{CONCLUSIONS}

Q fever is a polymorphic disease which may, in serious cases, mimic leptospirosis. Diagnosis may be difficult in low prevalence areas where the disease is not well known.

\section{REFERENCES}

1. Suárez Ortega S, Coello García I, Rodríguez Fernández JM, Arkuch Saade ME, Sanz Peláez O, Betancor León P. Miocarditis por Fiebre Q: presentación de un caso. Mapfre Med 2005;16:223-227.

2. Maurin M, Raoul D. Q fever. Clin Microbiol Rev 1999;12:518-553.

3. Suárez Ortega S, Rivero Vera J, Hemmersbach M, Artiles Campelo F, Reyes Pérez R. Betancor León P. Hepatitis colestásica grave por fiebre Q: presentación de un caso. Gastroenterol Hepatol 2010;33:21-24.

4. Anderson A, Bijlmer H, Fournier PE, Graves S, Hartzell J, Kersh GJ, et al. Diagnosis and manage-ment of Q fever--United States, 2013: recommendations from CDC and the Q Fever Working Group. MMWR Recomm Rep 2013;62(RR-03):1-30.

5. Somasundaram R, Loddenkemper C, Zeitz M, Schneider T. A souvenir from the Canary Islands. Lancet 2006;367:1116. 